Bundesgesundheitsbl 2020 63:439-451 https://doi.org/10.1007/s00103-020-03108-9 Online publiziert: 10 . März 2020

(c) Der/die Autor(en) 2020

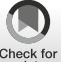

Lina Jaeschke ${ }^{1}$ Astrid Steinbrecher ${ }^{1} \cdot$ Karin Halina Greiser ${ }^{2}$ Marcus Dörr ${ }^{3,4}$. Thomas Buck $^{5}$. Jakob Linseisen ${ }^{6,7}$. Christa Meisinger ${ }^{6,7} \cdot$ Wolfgang Ahrens $^{8,9}$. Heiko Becher ${ }^{10} \cdot$ Klaus Berger $^{11}$ - Bettina Braun ${ }^{11,12} \cdot$ Hermann Brenner $^{13}$. Stefanie Castell ${ }^{14} \cdot$ Beate Fischer $^{15} \cdot$ Claus-Werner Franzke $^{16} \cdot$ Sylvia Gastell ${ }^{17}$. Kathrin Günther ${ }^{8}$. Wolfgang Hoffmann ${ }^{3}$. Bernd Holleczek ${ }^{18}$. Annika Jagodzinski $^{10} \cdot$ Rudolf Kaaks $^{2}$. Alexander Kluttig ${ }^{19} \cdot$ Gérard Krause $^{14,20}$. Lilian Krist ${ }^{21}$. Oliver Kuß ${ }^{22}$. Anna-Therese Lehnich ${ }^{23}$. Michael Leitzmann ${ }^{15}$. Wolfgang Lieb ${ }^{24} \cdot$ Markus Löffler $^{25} \cdot$ Karin B. Michels ${ }^{16} \cdot$ Rafael Mikolajczyk $^{19}$. Annette Peters ${ }^{26} \cdot$ Tamara Schikowski $^{27} \cdot$ Sabine Schipf ${ }^{3,28}$ - Börge Schmidt ${ }^{23}$. Matthias Schulze $^{29} \cdot$ Henry Völzke ${ }^{3,4,28} \cdot$ Stefan N. Willich ${ }^{21} \cdot$ Tobias Pischon $^{1,30,31,32}$

\title{
Erfassung selbst berichteter kardiovaskulärer und metabolischer Erkrankungen in der NAKO Gesundheitsstudie: Methoden und erste Ergebnisse
}

re und metabolische Erkrankungen sowie assoziierte Komplikationen und Operationen erfasst [3-5]. Dabei erhebt die NAKO nicht den Anspruch, unmittelbar Prävalenzen kardiovaskulärer und metabolischer Erkrankungen in Deutschland abzuschätzen. Es ist aber für geplante Forschungsvorhaben wichtig, die Breite des Spektrums der Erkrankungshäufigkeit in der NAKO zu kennen, um die Basispopulation zu charakterisieren und Vergleiche mit anderen Populationen zu ermöglichen. Aktuell liegen diese Daten für die ersten ca. 100.000 Teilnehmenden zur Halbzeit der Basiserhebung vor.

Ziel dieses Artikels ist einerseits, die Methoden zu beschreiben, mit denen selbst berichtete, ärztlich diagnostizierte kardiovaskuläre und metabolische Erkrankungen und Operationen in der NAKO-Basiserhebung erfasst wurden. Zudem soll eine Übersicht über deren Häufigkeit in den ersten ca. 100.000 Teilnehmenden gegeben werden. Die beobachteten Häufigkeiten werden im Kontext aktueller für Deutschland berichteter Zahlen eingeordnet und diskutiert.

\section{Methoden}

\section{Studienpopulation}

Die Daten wurden im Rahmen der NAKO erhoben, in deren Basiserhebung von März 2014 bis September 2019 in 18 bundesweit verteilten Studienzentren insgesamt 205.000 Teilnehmende aus der deutschen Allgemeinbevölkerung im Alter von 20-69 Jahren eingeschlossen wurden [4-6]. Die zufällige Ziehung der Bevölkerungsstichprobe erfolgte altersund geschlechtsstratifiziert (50\% Männer, 10-Jahres-Altersgruppen: je $10 \%$ in den zwei unteren, je $26,6 \%$ in den übrigen 10-Jahres-Altersgruppen) durch die jeweils zuständigen lokalen Melderegister [3, 4, 6]. Einschlusskriterien waren Wohnort im Einzugsgebiet des für das Studienzentrum verantwortlichen Einwohnermeldeamtes, Alter 20-69 Jahre, ausreichende Deutschkenntnisse und die Fähigkeit, eine informierte Einverständniserklärung abzugeben. Alle Teilnehmenden besuchten das jeweilige Studienzentrum und absolvierten umfangreiche Untersuchungen sowie Befragungen durch geschultes und zertifiziertes Studienpersonal zur Erhebung 
einer Vielzahl von Krankheiten und potenziellen Risikofaktoren. Die Befragungen erfolgten im Rahmen eines persönlichen, computergestützten Interviews sowie touchscreenbasiert (Selbstausfüller). Vorläufige Analysen weisen darauf hin, dass die Responseproportion in der NAKO bei etwa $18 \%$ liegt [6]. Details zum Studienprotokoll sind in der Publikation von Schipf et al. nachzulesen [6]. Im Jahr 2017 wurde ein „Data Freeze 100k“ nach den ersten 100.000 Teilnehmenden zur Qualitätskontrolle und ersten Beschreibung der Erhebung und der Daten beschlossen und mit Stichtag 17.03.2017 durchgeführt [6]. Auf dieser Datenbasis zur Halbzeit der Basiserhebung (Midterm Baseline Dataset) beruht die vorliegende Studie. Insgesamt wurde ein Datensatz mit 101.814 Teilnehmenden zur Verfügung gestellt. Davon wurden wegen fehlender Altersangabe 8 Teilnehmende ausgeschlossen und somit insgesamt 101.806 Teilnehmende in die folgenden Analysen eingeschlossen.

\section{Datenerhebung im Bereich kardiovaskulärer und meta- bolischer Erkrankungen}

Die Datenerhebung folgte einem für alle Studienzentren einheitlichen Standardprotokoll [6].

Im Interview wurden die Teilnehmenden gefragt, ob bei ihnen jemals ein Arzt eine der folgenden kardiovaskulären Erkrankungen diagnostiziert hat (Antwortmöglichkeiten: Ja, Nein, keine Angabe, weiß nicht): „Herzinfarkt“; „Verengung der Herzkranzgefäße bzw. Angina pectoris" (nachfolgend Angina pectoris); „Herzschwäche bzw. Herzinsuffizienz" (nachfolgend Herzinsuffizienz); „Herzrhythmusstörungen“; „Schaufensterkrankheit bzw. Durchblutungsstörungen in den Beinen, auch Claudicatio intermittens oder arterielle Verschlusskrankheit genannt" (nachfolgend Claudicatio intermittens); „Bluthochdruck“ (nachfolgend arterielle Hypertonie). Bezogen auf metabolische Erkrankungen wurden analog folgende Diagnosen abgefragt: „Zuckerkrankheit bzw. Diabetes mellitus" (nachfolgend Diabetes mellitus); „erhöhte Blutfette,
Cholesterin oder Triglyzeride“ (nachfolgend Hyperlipidämie); „Gicht oder eine andere Harnsäureerkrankung“ (nachfolgend Gicht); „Schilddrüsenerkrankung“.

Gaben Teilnehmende eine dieser kardiovaskulären oder metabolischen Diagnosen an, wurden sie nach Jahr oder alternativ Alter bei Erstdiagnose gefragt und ob sie diesbezüglich in den vorangegangenen 12 Monaten behandelt wurden (nicht bei Herzinfarkt und Diabetes). Bei berichtetem Herzinfarkt wurde nach Anzahl, Jahr bzw. Alter bei Infarkt sowie nach Art der Behandlung (gar nicht, ambulant in Praxis, stationär im Krankenhaus) vom ersten und gegebenenfalls bislang letzten Infarkt gefragt. Teilnehmende mit Diabetes wurden gefragt, ob sie mit „Insulin“, „Tabletten“ (nachfolgend orale Antidiabetika) oder beidem, „diätetisch“ oder „anderweitig“ behandelt werden (Ja, Nein, keine Angabe, weiß nicht). Bei berichteter Insulin- und/oder oraler Antidiabetikabehandlung wurden Jahr bzw. Alter bei Behandlungsbeginn erfragt, dies gegebenenfalls für beide Therapieformen. Darüber hinaus wurde erfragt, ob jemals diabetesbedingt eine der folgenden Komplikationen aufgetreten ist (Ja, Nein, keine Angabe, weiß nicht): „Retinopathie, d.h. Gefäßschäden am Augenhintergrund“; „Erblindung“; „Eiweiß im Urin“ (nachfolgend Albuminurie); „Nierenversagen oder Einschränkung der Nierenfunktion“ (nachfolgend Niereninsuffizienz); „Behandlung mit ,künstlicher Niere, also Dialyse oder Nierentransplantation" (nachfolgend Nierenersatztherapie); „Nervenleiden in den Beinen, z. B. Taubheit, Brennen, Kribbeln" (nachfolgend Polyneuropathie); „Amputationen, z. B. von Zeh, Fuß, Unter- oder Oberschenkel“; „diabetisches Fußsyndrom (schlecht heilendes Geschwür)“. Teilnehmerinnen wurden zudem gefragt, ob der Diabetes erstmals in einer Schwangerschaft diagnostiziert wurde (Ja, Nein, keine Angabe, weiß nicht). Teilnehmende mit Schilddrüsenerkrankung wurden gefragt, ob sie diesbezüglich unabhängig vom Zeitraum der letzten 12 Monate folgendermaßen behandelt wurden (Ja, Nein, keine Angabe, weiß nicht): „Schilddrüsenhormone“; „Radiojodthe- rapie“; „Bestrahlung im Halsbereich“; „Schilddrüsen-Operation“; „Jodtabletten“; „andere Behandlung“.

Zudem wurden Teilnehmende im Interview gefragt, ob bei ihnen jemals eine der folgenden Operationen durchgeführt wurde (Ja, Nein, keine Angabe, weiß nicht) und wenn Ja, in welchem Jahr bzw. Alter: „Herzklappen-Operation“; „Ballondilatation der Herzkranzgefäße (PTCA) mit/ohne Stent-Implantation“ (nachfolgend PTCA, perkutane transluminale Coronar-Angioplastie); „BypassOperation am Herzen“; „Schrittmacher-Implantation“; „Ballondilatation der Beinarterien mit/ohne Stent-Implantation“; „Bypass-Operation an den Beinarterien“; „Operation der Halsschlagader (Karotisarterie)“ (nachfolgend Karotisangioplastie); „vollständige oder teilweise Entfernung der (Neben-) Schilddrüse“ (nachfolgend Schilddrüsenresektion); „Herztransplantation“.

In der touchscreenbasierten Befragung wurden Teilnehmende $\geq 50$ Jahre zusätzlich gebeten anzugeben, ob bei ihnen Folgendes zutrifft (Ja, Nein, keine Angabe, weiß nicht): „abendlich geschwollene Beine“; „nachts regelmäßiges Aufstehen zum Wasserlassen“" (wenn Ja, wie oft in der Regel pro Nacht); „Luftnot und/oder Schwächegefühl bei körperlicher Belastung“ (wenn Ja, bei welcher Belastungsstärke: geringe, mittlere oder schwere Belastung); „Schlafen mit erhöhtem Oberkörper" (wenn Ja, Höhe des Oberkörpers: 15-30 Grad, 31-45 Grad oder $\geq 45$ Grad) [7].

\section{Vergleichsdaten für Deutschland}

Um die beobachteten Häufigkeiten kardiovaskulärer und metabolischer Erkrankungen und Operationen einzuordnen, wurden primär Vergleichsdaten herangezogen, die wie die NAKO auf Selbstangaben zu jemals erfolgten Diagnosen in epidemiologischen Studien beruhen. Ziel dabei war es, soweit möglich, Zahlen für das gesamte Bundesgebiet getrennt nach Geschlecht zu nutzen. Dies waren zum einen Daten des Robert Koch-Instituts (RKI) aus dem Befragungs- und Untersuchungssurvey „Studie zur Gesundheit Erwachsener in Deutschland“ (DEGS; [8]). Hier wurde die selbst berichtete 
Bundesgesundheitsbl 2020 ·63:439-451 https://doi.org/10.1007/s00103-020-03108-9

(c) Der/die Autor(en) 2020

L. Jaeschke · A. Steinbrecher · K. H. Greiser · M. Dörr · T. Buck · J. Linseisen · C. Meisinger · W. Ahrens · H. Becher · K. Berger · B. Braun · H. Brenner ·

S. Castell · B. Fischer · C.-W. Franzke · S. Gastell · K. Günther · W. Hoffmann · B. Holleczek · A. Jagodzinski · R. Kaaks · A. Kluttig · G. Krause · L. Krist · O. Kuß · A.-T. Lehnich · M. Leitzmann · W. Lieb · M. Löffler · K. B. Michels · R. Mikolajczyk · A. Peters · T. Schikowski · S. Schipf · B. Schmidt ·

M. Schulze $\cdot$ H. Völzke $\cdot$ S. N. Willich $\cdot$ T. Pischon

\section{Erfassung selbst berichteter kardiovaskulärer und metabolischer Erkrankungen in der NAKO Gesundheitsstudie: Methoden und erste Ergebnisse}

Zusammenfassung

Hintergrund. Aus der NAKO Gesundheitsstudie, der größten deutschen bevölkerungsbasierten Studie zur Gesundheit, liegen die Daten zu selbst berichteten kardiovaskulären und metabolischen Erkrankungen der ersten ca. 100.000 Teilnehmenden vor. Ziel. Beschreibung der Methoden zur Erhebung sowie Berechnung der Häufigkeit kardiovaskulärer und metabolischer Erkrankungen in der NAKO.

Material und Methoden. 101.806 Teilnehmende (20-75 Jahre, 46\% Männer) aus 18 bundesweiten Studienzentren wurden in einem computergestützten, standardisierten persönlichen Interview gebeten, anhand einer vorgegebenen Liste anzugeben, welche Erkrankungen bei ihnen jemals ärztlich diagnostiziert wurden, darunter auch kardiovaskuläre und metabolische Erkrankungen. Für Letztere wurden deren geschlechtsspezifische relative Häufigkeiten berechnet und diese mit Referenzzahlen verglichen.

Ergebnisse. Bezogen auf die kardiovaskulären Erkrankungen berichteten 3,5\% der Männer und $0,8 \%$ der Frauen, jemals einen Herzinfarkt diagnostiziert bekommen zu haben, 4,8 \% bzw. 1,5\% eine Angina pectoris, 3,5\% bzw. $2,5 \%$ eine Herzinsuffizienz, $10,1 \%$ bzw. $10,4 \%$ Herzrhythmusstörungen, $2,7 \%$ bzw. $1,8 \%$ eine Claudicatio intermittens und $34,6 \%$ bzw. $27,0 \%$ eine arterielle Hypertonie. Die Häufigkeit berichteter diagnostizierter metabolischer Erkrankungen lag bei 8,1\% bzw. 5,8 \% für Diabetes mellitus, bei 28,6\% bzw. 24,5\% für Hyperlipidämie, bei 7,9\% bzw. $2,4 \%$ für Gicht und bei $10,1 \%$ bzw. 34,3\% für Schilddrüsenerkrankungen. Die beobachteten Häufigkeiten lagen insgesamt etwas niedriger als Vergleichsdaten für Deutschland. Diskussion. Die NAKO erhebt von allen Teilnehmenden selbst berichtete, ärztlich diagnostizierte kardiovaskuläre und metabolische Erkrankungen und stellt damit eine Datenressource für weitergehende kardiometabolische Forschungsfragestellungen dar.

Schlüsselwörter

Epidemiologie $\cdot$ Kohorte $\cdot$ Beobachtungsstudie - Querschnittsstudie · Deutschland

\section{Assessment of self-reported cardiovascular and metabolic diseases in the German National Cohort (GNC, NAKO Gesundheitsstudie): methods and initial results}

\section{Abstract}

Background. Data on self-reported cardiovascular and metabolic diseases are available for the first 100,000 participants of the population-based German National Cohort (GNC, NAKO Gesundheitsstudie).

Objectives. To describe assessment methods and the frequency of self-reported cardiovascular and metabolic diseases in the German National Cohort.

Materials and methods. Using a computerbased, standardized personal interview, 101,806 participants ( $20-75$ years, $46 \%$ men) from 18 nationwide study centres were asked to use a predefined list to report medical conditions ever diagnosed by a physician, including cardiovascular or metabolic diseases. For the latter, we calculated sexstratified relative frequencies and compared these with reference data.

Results. With regard to cardiovascular diseases, $3.5 \%$ of men and $0.8 \%$ of women reported to have ever been diagnosed with a myocardial infarction, $4.8 \%$ and $1.5 \%$ with angina pectoris, $3.5 \%$ and $2.5 \%$ with heart failure, $10.1 \%$ and $10.4 \%$ with cardiac arrhythmia, $2.7 \%$ and $1.8 \%$ with claudicatio intermittens, and $34.6 \%$ and $27.0 \%$ with arterial hypertension. The frequencies of selfreported diagnosed metabolic diseases were $8.1 \%$ and $5.8 \%$ for diabetes mellitus, $28.6 \%$ and $24.5 \%$ for hyperlipidaemia, $7.9 \%$ and $2.4 \%$ for gout, and $10.1 \%$ and $34.3 \%$ for thyroid diseases. Observed disease frequencies were lower than reference data for Germany. Conclusions. In the German National Cohort, self-reported cardiovascular and metabolic diseases diagnosed by a physician are assessed from all participants, therefore representing a data source for future cardiometabolic research in this cohort.

Keywords

Epidemiology · Cohort · Observational study . Cross-sectional study · Germany
Häufigkeit von Herzinfarkt (Selbstangabe einer jemals erfolgten ärztlichen Diagnose), Angina pectoris (Selbstangabe einer jemals erfolgten ärztlichen Diagnose einer gestörten Blutzufuhr zum Herzen, Verengung der Herzkranzgefäße oder einer Angina pectoris), arterieller Hypertonie (hypertoner Blutdruckmesswert oder Einnahme antihypertensiver Medikamente in den letzten 7 Tagen bei bekannter Hypertonie), Diabetes melli- tus (Selbstangabe einer jemals erfolgten ärztlichen Diagnose oder Antidiabetikaeinnahme in den letzten 7 Tagen) sowie Dyslipidämie (Selbstangabe einer jemals erfolgten ärztlichen Diagnose einer Fettstoffwechselstörung) bei Teilnehmenden $\geq 20$ Jahre im Zeitraum 2008-2011 erhoben [9-13]. Der RKI-Telefonsurvey "Gesundheit in Deutschland aktuell“ (GEDA) aus 2012 bzw. 2014/2015 wurde ebenso herangezogen, wobei hier die 12-
Monats-Prävalenz selbst berichteter Diagnosen von Hypertonie, Diabetes und Hyperlipidämie (für Diabetes auch als Lebenszeitprävalenz) bei Teilnehmenden $\geq 18$ Jahren im Zeitraum 2012-2013 bzw. 2014-2015 erhoben wurde [14, 15]. In DEGS und GEDA wurden beobachtete Häufigkeiten altersstandardisiert berichtet (auf Basis von Zahlen des Statistischen Bundesamtes 2010 bzw. der „alten Europastandardbevölkerung“; in 
Tab. 1 Eingeschlossene Teilnehmende in der NAKO Gesundheitsstudie zur Halbzeit der Basiserhebung nach Studienzentrum

\begin{tabular}{|c|c|c|c|c|c|c|}
\hline & \multicolumn{2}{|l|}{ Gesamt } & \multicolumn{2}{|c|}{ Männer } & \multicolumn{2}{|l|}{ Frauen } \\
\hline & $N$ & $\%$ & $n$ & $\%$ & $n$ & $\%$ \\
\hline Studienzentrum & 101.806 & 100,0 & 47.266 & 100,0 & 54.540 & 100,0 \\
\hline Augsburg & 9927 & 9,8 & 4879 & 10,3 & 5048 & 9,3 \\
\hline Berlin-Mitte & 6588 & 6,5 & 2896 & 6,1 & 3692 & 6,8 \\
\hline Berlin-Nord & 5503 & 5,4 & 2471 & 5,2 & 3032 & 5,6 \\
\hline Berlin-Süd & 5138 & 5,0 & 2247 & 4,8 & 2891 & 5,3 \\
\hline Bremen & 6370 & 6,3 & 2726 & 5,8 & 3644 & 6,7 \\
\hline Düsseldorf & 3282 & 3,2 & 1517 & 3,2 & 1765 & 3,2 \\
\hline Essen & 4159 & 4,1 & 1951 & 4,1 & 2208 & 4,0 \\
\hline Freiburg & 4578 & 4,5 & 2146 & 4,5 & 2432 & 4,5 \\
\hline Halle & 5186 & 5,1 & 2426 & 5,1 & 2760 & 5,1 \\
\hline Hamburg & 4007 & 3,9 & 1920 & 4,1 & 2087 & 3,8 \\
\hline Hannover & 5578 & 5,5 & 2504 & 5,3 & 3074 & 5,6 \\
\hline Kiel & 4109 & 4,0 & 1879 & 4,0 & 2230 & 4,1 \\
\hline Leipzig & 7448 & 7,3 & 3440 & 7,3 & 4008 & 7,3 \\
\hline Mannheim & 3990 & 3,9 & 1854 & 3,9 & 2136 & 3,9 \\
\hline Münster & 5042 & 5,0 & 2479 & 5,2 & 2563 & 4,7 \\
\hline Neubrandenburg & 11.859 & 11,6 & 5753 & 12,2 & 6106 & 11,2 \\
\hline Regensburg & 4320 & 4,2 & 2102 & 4,4 & 2218 & 4,1 \\
\hline Saarbrücken & 4722 & 4,6 & 2076 & 4,4 & 2646 & 4,9 \\
\hline
\end{tabular}

DEGS zusätzlich gewichtet; $[8,14,16])$. Lagen keine Zahlen für Gesamtdeutschland vor, wurden regionale epidemiologische Studien herangezogen, wie die für Nordostdeutschland repräsentative Studie Study of Health in Pomerania (SHIP; [17]) oder die Gutenberg-Gesundheitsstudie der Rhein-Main-Region [18].

Wenn keine Vergleichsdaten aus epidemiologischen Studien zur Verfügung standen, wurden auf Abrechnungs- und Routinedaten beruhende Schätzungen herangezogen. So wurde die Häufigkeit der Herzinsuffizienz, Herzrhythmusstörungen, Herzklappenoperation, Bypassoperation am Herzen und Schrittmacherimplantation mit Angaben des Deutschen Herzberichts 2018, der auf der Krankenhausdiagnosestatistik des Statistischen Bundesamtes abgeleitet aus der altersstandardisierten vollstationären Hospitalisationsrate (alle Alter) für das Jahr 2017 basiert, verglichen [19]. Für die Herzinsuffizienz wurden auch Schätzungen basierend auf Krankenkassendaten herangezogen [20], für die Claudicatio intermittens die Gesundheitsberichterstattung des Bundes (GBE;
1998), die auf Zahlen unter anderem des Statistischen Bundesamtes beruht [21]. Schätzungen zur Häufigkeit diabetesbedingter Komplikationen wurden dem Gesundheitsbericht 2019 der Deutschen Diabetes Gesellschaft entnommen, der auf populationsbezogenen Studien und Krankenkassendaten beruht [22], und zur Gichthäufigkeit Angaben der Deutschen Rheuma- und Gicht-Liga [23, 24]. Zur Häufigkeit der Schilddrüsenresektion liegen Zahlen vom Statistischen Bundesamt basierend auf der fallpauschalenbezogenen Krankenhausstatistik 2017 vor [25].

\section{Statistische Analysen}

Für die Häufigkeitsbeschreibung der berichteten Selbstangaben zu Erkrankungen, Operationen und Behandlungsformen wurden die relativen Anteile bezogen auf die jeweilige Stichprobe (Gesamtstichprobe bzw. Stichprobe mit entsprechend berichteter Diagnose) berechnet. Neben den rohen (beobachteten) wurden auch die direkt altersstandardisierten Häufigkeiten zur Vergleichbarkeit mit den genannten Vergleichsdaten auf
Basis der Zahlen des Statistischen Bundesamtes 2011 sowie der „alten Europastandardbevölkerung" (definiert von der Weltgesundheitsorganisation im Jahr 1976) unter Einbeziehung der relevanten Altersgruppen berechnet [26, 27].

Aus den berichteten Jahres- bzw. Altersangaben zum Zeitpunkt der Erstdiagnose einer Erkrankung oder Operation wurde einheitlich das Alter bei Erstdiagnose bzw. Operation berechnet. Die Fallzahlen, auf denen diese Auswertung beruht, ergeben sich aus $\bullet$ Tab. 2 für kardiovaskuläre und aus $\bullet$ Tab. 3 für metabolische Erkrankungen, aus - Tab. 6 für die kardiologischen, gefäßchirurgischen Operationen und Schilddrüsenresektionen. Zum Alter bei Erstdiagnose Herzinfarkt, Angina pectoris, Herzinsuffizienz, Herzrhythmusstörungen, Claudicatio intermittens und arterielle Hypertonie lagen für $2,6 \%, 2,7 \%, 2,6 \%$, 2,4\%, 2,4\% bzw. 1,8\% der Teilnehmenden keine Informationen vor (d.h. , keine Angabe“ oder "weiß nicht" bei Jahresoder Altersvariable oder fehlende Angaben in beiden Variablen), bei Diabetes mellitus, Hyperlipidämie, Gicht und Schilddrüsenerkrankung für $1,0 \%, 5,3 \%$, 2,6\% bzw. 2,4\%, bei Herzklappenoperation, PTCA, Bypassoperation am Herzen, Schrittmacherimplantation, Ballondilatation der Beinarterien, Bypassoperation an den Beinarterien, Karotisangioplastie und Schilddrüsenresektion für $0,5 \%, 0,9 \%, 0,3 \%, 0,0 \%, 1,4 \%, 2,0 \%$, $0,4 \%$, bzw. $1,0 \%$. Das Alter bei Erstdiagnose Angina pectoris, Herzinsuffizienz, Herzrhythmusstörungen und arterielle Hypertonie gaben $0,06 \%, 0,7 \%$, 0,3\% bzw. 0,003\% der Teilnehmenden mit 0 Jahren an, für Diabetes, Hyperlipidämie und Schilddrüsenerkrankung $0,03 \%, 0,01 \%$ bzw. $0,03 \%$, bezogen auf Herzklappenoperationen $0,3 \%$.

Die Häufigkeit des berichteten Alters bei Erstdiagnose bzw. Operation wurde grafisch in den vier Strata $<40,40-49$, $50-59$ und $\geq 60$ Jahre dargestellt. Teilnehmende mit fehlendem oder selbst berichtetem Alter bei Erstdiagnose von 0 Jahren wurden hierbei ausgeschlossen. Analog wurden für den Diabetes Alter bei Beginn und Dauer der Behandlung berechnet. Diese wurden als Median mit Interquartilsabstand (Interquartile 
Tab. 2 Häufigkeiten von selbst berichteten, ärztlich diagnostizierten kardiovaskulären Erkrankungen und Anzahl Herzinfarktein derNAKO zurHalbzeit der Basiserhebung

\begin{tabular}{|c|c|c|c|c|c|c|c|c|c|c|}
\hline & \multicolumn{5}{|c|}{ Männer ( $(n=47.266)$} & \multicolumn{5}{|c|}{ Frauen $(n=54.540)$} \\
\hline & \multicolumn{4}{|c|}{ NAKO } & \multirow{2}{*}{$\begin{array}{l}\text { Vergleichs- } \\
\text { daten } \\
\%\end{array}$} & \multicolumn{4}{|c|}{ NAKO } & \multirow{2}{*}{$\begin{array}{l}\text { Vergleichs- } \\
\text { daten } \\
\%\end{array}$} \\
\hline & $n$ & $\%$, roh & $\begin{array}{l}\%, \\
\text { stand. }^{a}\end{array}$ & $\begin{array}{l}\%, \\
\text { stand. }^{b}\end{array}$ & & $n$ & $\%$, roh & $\begin{array}{l}\%, \\
\text { stand. }^{a}\end{array}$ & $\begin{array}{l}\text { \%, } \\
\text { stand. }^{b}\end{array}$ & \\
\hline Herzinfarkt & 1631 & 3,5 & 2,4 & 2,0 & $\begin{array}{l}7,0[9] / 5,6 \\
{[19]^{\mathrm{d}}}\end{array}$ & 415 & 0,8 & 0,6 & 0,5 & $\begin{array}{l}2,5[9] / 1,7 \\
{[19]^{\mathrm{d}}}\end{array}$ \\
\hline \multicolumn{11}{|l|}{ Wenn Ja, Anzahl Herzinfarkte ${ }^{c}$} \\
\hline 1 & 1344 & 82,4 & - & - & - & 364 & 87,7 & - & - & - \\
\hline 2 & 205 & 12,6 & - & - & - & 38 & 9,2 & - & - & - \\
\hline$>2$ & 79 & 4,8 & - & - & - & 9 & 2,2 & - & - & - \\
\hline Angina pectoris & 2269 & 4,8 & 3,4 & 2,8 & $\begin{array}{l}10,4[9] / 3,8 \\
{[19]^{d}}\end{array}$ & 836 & 1,5 & 1,3 & 1,0 & $\begin{array}{l}5,7[9] / 2,0 \\
{[19]^{d}}\end{array}$ \\
\hline Herzinsuffizienz & 1671 & 3,5 & 2,7 & 2,3 & $\begin{array}{l}1,9[20] / 5,6 \\
{[19]^{\mathrm{d}}}\end{array}$ & 1339 & 2,5 & 2,1 & 1,7 & $\begin{array}{l}1,6[20] / 5,6 \\
{[19]^{d}}\end{array}$ \\
\hline Herzrhythmusstörung & 4754 & 10,1 & 7,9 & 7,1 & $6,3[19]^{d}$ & 5649 & 10,4 & 8,7 & 8,0 & $5,4[19]^{d}$ \\
\hline Claudicatio intermittens & 1282 & 2,7 & 1,9 & 1,6 & $4,0[21]^{\mathrm{e}}$ & 968 & 1,8 & 1,5 & 1,3 & $4,0[21]^{\mathrm{e}}$ \\
\hline Arterielle Hypertonie & 16.355 & 34,6 & 25,7 & 23,3 & $\begin{array}{l}33,3 \\
{[10] / 28,5} \\
{[14]^{f}}\end{array}$ & 14.736 & 27,0 & 21,2 & 18,4 & $\begin{array}{l}29,9 \\
{[10] / 28,2} \\
{[14]^{f}}\end{array}$ \\
\hline $\begin{array}{l}\text { Mindestens eine der genann- } \\
\text { ten kardiovaskulären Erkran- } \\
\text { kungen }\end{array}$ & 19.685 & 41,6 & 31,5 & 28,7 & $--^{9}$ & 18.692 & 34,3 & 27,5 & 24,5 & $--^{9}$ \\
\hline \multicolumn{11}{|c|}{ 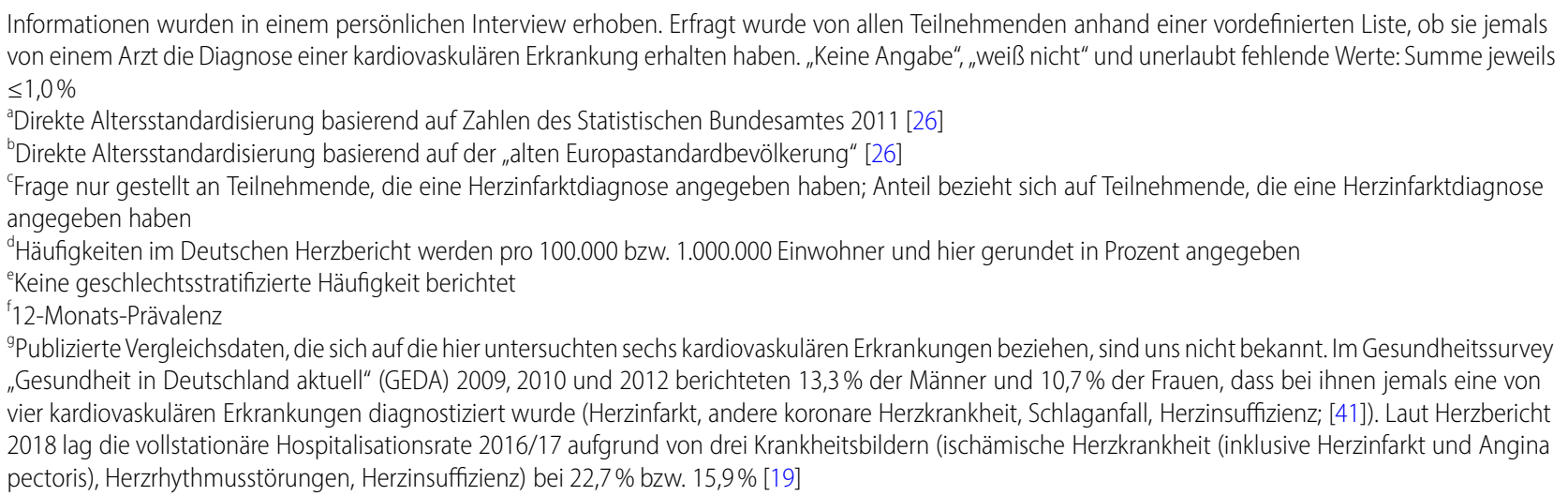 } \\
\hline
\end{tabular}

Range, IQR) dargestellt; Teilnehmende mit Altersangabe bei Therapiebeginn 0 Jahre $(0,1 \%$ für Insulin) wurden dafür ausgeschlossen.

Alle Analysen wurden mit SAS ${ }^{\circledR}$ Enterprise Guide ${ }^{\circledR}$ (Version 4.3; SAS Institute Inc., Cary, NC, USA) durchgeführt.

\section{Ergebnisse}

Insgesamt wurden 101.806 Teilnehmende (46,4\% Männer) in die Analysen eingeschlossen (- Tab. 1). Das mittlere Alter lag bei den Männern bei 52,5 Jahren (Standardabweichung 12,4) und bei den Frauen bei 51,5 Jahren $(12,4)$.

\section{Kardiovaskuläre Erkrankungen}

Im Vergleich mit für Deutschland bekannten Schätzungen liegen die hier beobachteten standardisierten Häufigkeiten der Teilnehmenden, die berichteten, jemals im Laufe ihres Lebens von einem Arzt die Diagnose einer kardiovaskulären Erkrankung erhalten zu haben, etwas niedriger (•Tab. 2). So lag die auf Selbstangaben beruhende standardisierte und gewichtete Herzinfarktlebenszeitprävalenz in DEGS bei Männern bei 7,0\% und bei Frauen bei 2,5\% [9], während die Häufigkeit in der NAKO standardisiert (Statistisches Bundesamt 2011/,alte Europastandardbevölkerung“
[26]) bei 2,4/2,0\% bzw. 0,6/0,5\% lag. Die standardisierte Häufigkeit von Herzrhythmusstörungen lag in der NAKO mit 7,9/7,1\% bzw. 8,7/8,0\% hingegen höher als die Angaben im Deutschen Herzbericht mit 6,3\% bzw. 5,4\% auf Basis der Krankenhausdiagnosestatistik (im Herzbericht pro 100.000 bzw. 1.000.000 Einwohner und hier gerundet in \% angegeben; [19]).

Teilnehmende mit Selbstangabe eines Herzinfarkts wurden gefragt, in welchem Rahmen sie behandelt wurden. 2,5\% der Männer bzw. 5,1 \% der Frauen gaben an, beim ersten bzw. bislang einzigen Infarkt ambulant behandelt worden zu sein (beim bislang letzten bei $\geq 2$ Infarkten: 


\section{Leitthema}
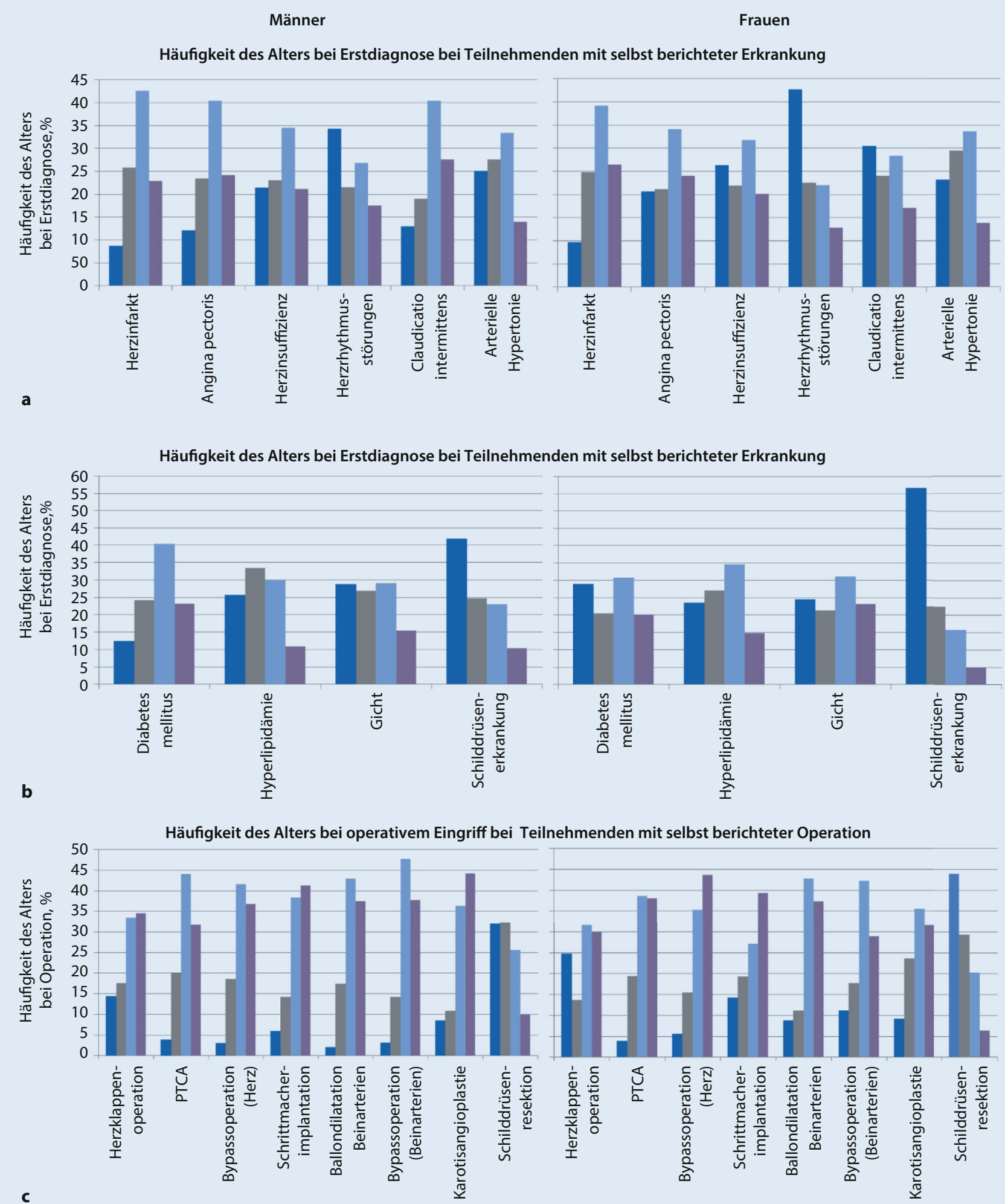
Abb. 14 Häufigkeit des selbst berichteten Alters $<40,40-49,50-59$ und $\geq 60$ Jahren bei der Erstdiagnose einer laut Selbstbericht jemals ärztlich diagnostizierten kardiovaskulären (a) oder metabolischen Erkrankung (b) bzw. bei einer laut Selbstbericht jemals durchgeführten kardiologischen oder gefäßchirurgischen Operation sowie Schilddrüsenresektion (c) bei Männern und Frauen

$3,6 \%$ bzw. $4,3 \%$ ), $86,5 \%$ bzw. $80,5 \%$ stationär (91,1 \% bzw. 76,1\%). Laut Selbstangabe wurden $11,0 \%$ bzw. 14,2\% (5,3\% bzw. 19,6\%) gar nicht behandelt.

Bei Teilnehmenden, die berichteten, dass bei ihnen jemals eine kardiovaskuläre Erkrankung diagnostiziert wurde, lag der Häufigkeitsgipfel des selbst berichteten Alters bei Erstdiagnose zwischen 50-59 Jahren (• Abb. 1a). Ausnahme waren bei beiden Geschlechtern Herzrhythmusstörungen und Claudicatio intermittens bei Frauen, für die der Häufigkeitsgipfel jeweils im Stratum $<40$ Jahre lag.

54,2\% der Männer und 48,7\% der Frauen mit berichteter Angina-pectorisDiagnose gaben an, in den vorangegangenen 12 Monaten diesbezüglich behandelt worden zu sein. Bei Herzinsuffizienz waren es entsprechend $60,2 \%$ bzw. 50,6\%, bei Herzrhythmusstörungen $38,0 \%$ bzw. $30,9 \%$, bei Claudicatio intermittens $48,7 \%$ bzw. $37,4 \%$ und bei arterieller Hypertonie 77,0 \% bzw. 80,7\%.

\section{Metabolische Erkrankungen}

Verglichen mit bekannten Zahlen, lagen die in der NAKO-Stichprobe beobachteten standardisierten Häufigkeiten selbst berichteter metabolischer Erkrankungen tendenziell etwas niedriger (• Tab. 3). So schätzen DEGS und GEDA basierend auf Selbstangaben die standardisierte (DEGS: Statistisches Bundesamt 2010, zusätzliche Gewichtung; GEDA: „alte Europastandardbevölkerung") Lebenszeit- bzw. 12-Monats-Prävalenz von Diabetes auf 7,0-8,7 \% bei Männern und auf $7,4-9,0 \%$ bei Frauen [12, 28, 29], während sie in der NAKO standardisiert (Statistisches Bundesamt 2011/,,alte Europastandardbevölkerung") bei 5,6/4,9\% bzw. 4,9/4,4\% lag. Während die Deutsche Gicht-Liga von einer Gichthäufigkeit bei den 30 - bis 59 -Jährigen von $2,8 \%$ bzw. $0,4 \%$ ausgeht [23], lagen die NAKO-Zah- len mit 5,6/5,0 \% bzw. 1,9/1,6\% hingegen höher.

Bei Teilnehmenden mit berichteter metabolischer Erkrankung lag der Häufigkeitsgipfel des selbst berichteten Alters bei Erstdiagnose zwischen 50-59 Jahren (• Abb. 1b). Ausnahme waren Hyperlipidämie bei Männern und Schilddrüsenerkrankungen bei beiden Geschlechtern, für die der Häufigkeitsgipfel im Stratum 40-49 Jahre bzw. <40 Jahre lag.

39,6\% der Männer und 28,8\% der Frauen mit berichteter Hyperlipidämiediagnose gaben an, in den vorangegangenen 12 Monaten diesbezüglich behandelt worden zu sein. Bei Gicht waren es entsprechend 50,0\% bzw. 37,2\% und bei Schilddrüsenerkrankungen $57,9 \%$ bzw. $66,0 \%$.

Bezogen auf die berichtete Diabetestherapie (• Tab. 3) lag das mediane Alter bei Beginn einer alleinigen Insulintherapie bei 45 Jahren (Männer, IQR 31; 45) und 42 Jahren (Frauen, 25; 53). Bei alleiniger oraler Antidiabetikabehandlung lag es bei 55 Jahren (Männer, 50; 61) bzw. ebenfalls 55 Jahren (Frauen, 49; 61). Das mediane Alter zu Beginn einer kombinierten Therapie lag für Insulin bei 56 Jahren (Männer, 50; 62) und für orale Antidiabetika bei 50 Jahren (Männer, 44; 56), bei Frauen lag es entsprechend bei 55 Jahren $(47 ; 61)$ bzw. 48 Jahren $(42 ; 55)$. Die mediane Dauer der alleinigen Insulintherapie lag bei 12 Jahren (Männer, 5; 21) und bei 14 Jahren (Frauen, 6; 24), die der alleinigen oralen Antidiabetikatherapie lag bei 6 Jahren (Männer, 3; 10) bzw. 5 Jahren (Frauen, 2; 10). Die mediane Dauer bei kombinierter Behandlung betrug für Insulin 5 Jahre (Männer, 2; 10) und für orale Antidiabetika 12 Jahre (Männer, 6; 17) und entsprechend für Frauen 5 Jahre $(2 ; 12)$ bzw. 12 Jahre (7; 18).

Vergleichsdaten für die beobachteten Häufigkeiten selbst berichteter diabetesbedingter Komplikationen bei Personen mit Diabetes (• Tab. 4) liefert zum Beispiel der Deutsche Diabetesbericht. In diesem wird die Häufigkeit einer Polyneuropathie bei Diabetikern für beide Geschlechter zusammen mit 13-26\% angegeben (NAKO, roh: 22,1\% bei Männern bzw. 16,9\% bei Frauen), die eines diabetischen Fußsyndroms mit
2-10\% [22], dessen Häufigkeit (roh) in der NAKO bei 3,6\% bei Männern bzw. 2,1\% bei Frauen lag.

Die häufigste (alleinig oder in Kombination) genannte Behandlungsform bei Männern und Frauen mit Schilddrüsenerkrankung waren Schilddrüsenhormone (79,1\% bzw. 87,6\%; - Tab. 5).

Basierend auf den rohen Häufigkeitsschätzungen berichteten $27,1 \%$ der Männer und $31,9 \%$ der Frauen genau eine, $16,0 \%$ bzw. $17,7 \%$ zwei und $14,4 \%$ bzw. 12,3\% drei oder mehr der abgefragten kardiovaskulären oder metabolischen Erkrankungen.

\section{Kardiologische und gefäß- chirurgische Operationen sowie Schilddrüsenresektionen}

Vergleichsdaten für die beobachtete Häufigkeit selbst berichteter, jemals durchgeführter kardiologischer und gefäßchirurgischer Operationen sowie Schilddrüsenresektionen (• Tab. 6) stammen unter anderem aus dem Deutschen Herzbericht basierend auf der Krankenhausdiagnosestatistik. Nach diesem wurden im Jahr 2017 rund 180.000 herzchirurgische Eingriffe durchgeführt, von denen Bypass-/koronarchirurgische Operationen (0,58 \% der Bevölkerung) und Herzklappenoperationen $(0,42 \%)$ den größten Anteil ausmachten [19]. Eine Bypassoperation am Herzen berichteten in der NAKO standardisiert (Statistisches Bundesamt 2011/, alte Europastandardbevölkerung") 0,8/0,7 \% der Männer bzw. 0,1/0,1\% der Frauen, eine Herzklappenoperation entsprechend $0,5 / 0,4 \%$ bzw. $0,2 / 0,2 \%$.

Bei Teilnehmenden mit selbst berichteter Operation lag der Häufigkeitsgipfel des angegebenen Alters bei Operation in den Strata 50-59 Jahre und älter, mit Ausnahme von Schilddrüsenresektionen, die in den jüngeren Strata (<50 Jahre) häufiger waren (• Abb. 1c).

\section{Diskussion}

Die vorliegende Arbeit beschreibt die Erhebungsmethoden selbst berichteter, ärztlich diagnostizierter kardiovaskulärer und metabolischer Erkrankungen und Operationen in der NAKO-Basis- 
Tab. 3 Häufigkeiten von selbst berichteten, ärztlich diagnostizierten metabolischen Erkrankungen und Art der Diabetesbehandlung in der NAKO zur Halbzeit der Basiserhebung

\begin{tabular}{|c|c|c|c|c|c|c|c|c|c|c|}
\hline & \multicolumn{5}{|c|}{ Männer $(n=47.266)$} & \multicolumn{5}{|c|}{ Frauen $(n=54.540)$} \\
\hline & \multicolumn{4}{|c|}{ NAKO } & \multirow{2}{*}{$\begin{array}{l}\text { Vergleichsdaten } \\
\%\end{array}$} & \multicolumn{4}{|c|}{ NAKO } & \multirow{2}{*}{$\begin{array}{l}\text { Vergleichsdaten } \\
\%\end{array}$} \\
\hline & $n$ & $\begin{array}{l}\%, \\
\text { roh }\end{array}$ & $\begin{array}{l}\%, \\
\text { stand. }^{a}\end{array}$ & $\begin{array}{l}\text { \%, } \\
\text { stand. }^{\text {b }}\end{array}$ & & $n$ & $\begin{array}{l}\%, \\
\text { roh }\end{array}$ & $\begin{array}{l}\%, \\
\text { stand. }^{a}\end{array}$ & $\begin{array}{l}\%, \\
\text { stand. }^{b}\end{array}$ & \\
\hline Diabetes mellitus & 3838 & 8,1 & 5,6 & 4,9 & $\begin{array}{l}7,0[12] / 8,7 \% \\
{[14] / 8,6[29]^{\mathrm{e}}}\end{array}$ & 3163 & 5,8 & 4,9 & 4,4 & $\begin{array}{l}7,4[12] / 9,0 \% \\
{[14] / 7,0[29]^{\mathrm{e}}}\end{array}$ \\
\hline \multicolumn{11}{|l|}{ Wenn Ja, Art der Behandlung ${ }^{c}$} \\
\hline Nur Insulin & 453 & 11,8 & - & - & - & 288 & 9,1 & - & - & - \\
\hline Nur orale Antidiabetika & 2005 & 52,2 & - & - & - & 1272 & 40,2 & - & - & - \\
\hline $\begin{array}{l}\text { Insulin und orale Antidiabe- } \\
\text { tika }\end{array}$ & 655 & 17,1 & - & - & - & 373 & 11,8 & - & - & - \\
\hline Diätetisch & 449 & 11,7 & - & - & - & 608 & 19,2 & - & - & - \\
\hline Andere Behandlung & 171 & 4,5 & - & - & - & 322 & 10,2 & - & - & - \\
\hline \multicolumn{11}{|c|}{ Wenn Ja, Schwangerschaftsdiabetes $^{d}$} \\
\hline Ja & - & - & - & - & - & 659 & 20,8 & - & - & $5,9[22]$ \\
\hline Hyperlipidämie & 13.519 & 28,6 & 21,4 & 19,4 & $\begin{array}{l}28,1[13] / 20,2 \\
{[14]}\end{array}$ & 13.350 & 24,5 & 19,4 & 17,2 & $\begin{array}{l}27,1[13] / 19,7 \\
{[14]}\end{array}$ \\
\hline Gicht & 3711 & 7,9 & 5,6 & 5,0 & $2,8[23]^{\mathrm{f}} /-[24]^{\mathrm{g}}$ & 1290 & 2,4 & 1,9 & 1,6 & $0,4[23]^{f} /-[24]^{g}$ \\
\hline Schilddrüsenerkrankung & 4779 & 10,1 & 8,3 & 7,9 & $\begin{array}{l}35,9 \text { bzw. } 20,2 \\
{[39]^{\mathrm{h}}}\end{array}$ & 18.706 & 34,3 & 29,2 & 28,4 & $\begin{array}{l}35,9 \text { bzw. } 20,2 \\
{[39]^{\mathrm{h}}}\end{array}$ \\
\hline $\begin{array}{l}\text { Mindestens eine der ge- } \\
\text { nannten metabolischen } \\
\text { Erkrankungen }\end{array}$ & 19.157 & 40,5 & 31,5 & 28,7 & - & 27.298 & 50,1 & 27,5 & 24,5 & - \\
\hline \multicolumn{11}{|c|}{ 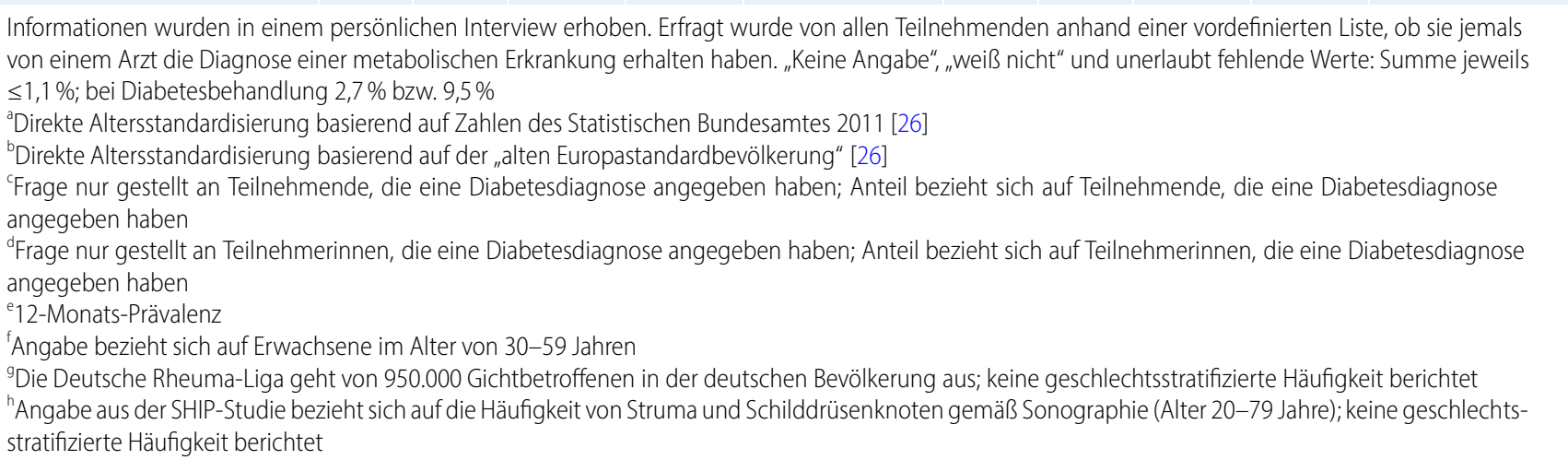 } \\
\hline
\end{tabular}

erhebung sowie deren Häufigkeit bei den ersten 101.806 Teilnehmenden. Die häufigste selbst berichtete kardiovaskuläre Erkrankung war bei Männern und Frauen die arterielle Hypertonie (34,6\% bzw. 27,0\%), die häufigste metabolische Erkrankung die Hyperlipidämie bei Männern (28,6\%) und Schilddrüsenerkrankungen bei Frauen (34,3\%). Die häufigste Operation war bei Männern die PTCA $(4,1 \%)$ und bei Frauen die Schilddrüsenresektion $(6,7 \%)$.

Die hier ausgewerteten Daten stammen aus der NAKO-Basiserhebung und liefern eine wichtige Grundlage für $\mathrm{zu}$ künftige wissenschaftliche Arbeiten, da sie die NAKO-Population im Hinblick auf die Häufigkeit kardiovaskulärer und metabolischer Erkrankungen sowie kardiologischer, gefäßchirurgischer Operationen und Schilddrüsenresektionen charakterisieren und stratumspezifische Auswertungen und Querschnittsanalysen ermöglichen. Darüber hinaus bilden sie die Basis zukünftiger Kohortenanalysen, in denen je nach Fragestellung meist Personen mit bestehenden (prävalenten) Krankheiten ausgeschlossen werden. Im Gegensatz dazu erfolgt die zukünftige Erfassung inzidenter Fälle in der NAKO über aktive und passive FollowUp-Prozeduren. Dazu werden den Teilnehmenden alle 2-3 Jahre Fragebögen $\mathrm{zu}$ inzidenten Krankheiten zugesen- det, die anschließend verifiziert werden. Außerdem werden Mortalität und Todesursachen erfasst und Abgleiche mit Krankenkassendaten implementiert.

Daten zur Häufigkeit selbst berichteter kardiovaskulärer und metabolischer Erkrankungen und Operationen aus epidemiologischen Studien liegen für Deutschland mit DEGS und GEDA vor. DEGS als Befragungs- und Untersuchungssurvey hat kardiovaskuläre und metabolische Erkrankungen wie die NAKO als Lebenszeitprävalenz aus Selbstangaben erhoben und setzt neben der Befragung teils noch auf klinische Untersuchungen wie Blutdruckmessungen oder Blutanalysen zur Unterscheidung bekannter 
Tab. 4 Häufigkeiten selbst berichteter diabetesbedingter Komplikationen bei Teilnehmenden mit berichteter ärztlicher Diabetesdiagnose in der NAKO zur Halbzeit der Basiserhebung ${ }^{\text {a }}$

\begin{tabular}{|c|c|c|c|c|c|c|}
\hline & \multicolumn{2}{|c|}{$\begin{array}{l}\text { Männer } \\
(n=3838)\end{array}$} & \multirow{2}{*}{$\begin{array}{l}\text { Vergleichs- } \\
\text { daten } \\
\%\end{array}$} & \multicolumn{2}{|c|}{ Frauen $(n=3163)$} & \multirow{2}{*}{$\begin{array}{l}\text { Vergleichs- } \\
\text { daten } \\
\%\end{array}$} \\
\hline & $n$ & $\%$ & & $n$ & $\%$ & \\
\hline Retinopathie & 237 & 6,2 & $22,6[18]$ & 130 & 4,1 & $21,1[18]$ \\
\hline Erblindung & 10 & 0,3 & $-[22]^{b c}$ & 10 & 0,3 & $-[22]^{b c}$ \\
\hline Proteinurie & 280 & 7,3 & $42[22]^{c d}$ & 299 & 9,5 & $42[22]^{c d}$ \\
\hline Niereninsuffizienz & 169 & 4,4 & - & 111 & 3,5 & - \\
\hline Nierenersatztherapie & 8 & 0,2 & - & 8 & 0,3 & - \\
\hline Polyneuropathie & 848 & 22,1 & $13-26[22]^{\mathrm{ce}}$ & 535 & 16,9 & $13-26[22]^{\text {ce }}$ \\
\hline Amputationen & 35 & 0,9 & $-[22]^{\mathrm{cf}}$ & 10 & 0,3 & $-[22]^{c f}$ \\
\hline $\begin{array}{l}\text { Diabetisches Fußsyn- } \\
\text { drom }\end{array}$ & 140 & 3,6 & $2-10[22]^{c}$ & 66 & 2,1 & $2-10[22]^{c}$ \\
\hline \multicolumn{7}{|c|}{$\begin{array}{l}\text { Informationen wurden in einem persönlichen Interview erhoben. Erfragt wurde von Teilnehmenden } \\
\text { mit Diabetesdiagnose anhand einer vordefinierten Liste, ob bei ihnen jemals aufgrund der Diabe- } \\
\text { teserkrankung eine der Komplikationen aufgetreten ist. „Keine Angabe“, „,weiß nicht“" und unerlaubt } \\
\text { fehlende Werte: Summe jeweils } \leq 1,8 \% \text {; bei Proteinurie } 6,8 \% \text { bzw. 6,1\% } \\
\text { 'Fragen nur gestellt an Teilnehmende, die eine Diabetesdiagnose angegeben haben. Anteil bezogen } \\
\text { auf Teilnehmende mit berichteter Diabetesdiagnose. Mehrfachnennungen möglich } \\
\text { 'Die Deutsche Diabetes Gesellschaft geht für Deutschland von } 2000 \text { Diabetespatienten aus, die von } \\
\text { Erblindung betroffen sind } \\
{ }^{c} \text { Keine geschlechtsstratifizierten Häufigkeiten berichtet } \\
\text { 'Angabe bezieht sich auf Nierenschäden, definiert als Mikroalbuminurie und/oder deutliche Nieren- } \\
\text { funktionseinschränkung; Angabe bezieht sich nur auf Typ-2-Diabetiker } \\
\text { "Angabe bezieht sich auf distalsymmetrische sensomotorische Polyneuropathien als häufigste und } \\
\text { klinisch bedeutsamste der diabetischen Neuropathien } \\
\text { 'Die Deutsche Diabetes Gesellschaft geht für Deutschland von } 8500 \text { hohen bzw. } 30.000 \text { Minoram- } \\
\text { putationen pro Jahr bei Patienten mit Diabetes aus }\end{array}$} \\
\hline
\end{tabular}

Tab. 5 Häufigkeiten der selbst berichteten Behandlungsarten bei Teilnehmenden mit berichteterärztlicher Diagnose einer Schilddrüsenerkrankung in der NAKO zur Halbzeit der Basiserhebung ${ }^{\mathrm{a}}$

\begin{tabular}{|l|l|l|l|l|} 
& \multicolumn{2}{l}{ Männer ( $\boldsymbol{n}=\mathbf{4 7 7 9})$} & \multicolumn{2}{l}{ Frauen ( $\boldsymbol{n}=\mathbf{1 8 . 7 0 6})$} \\
\hline & $\boldsymbol{n}$ & \% & $\boldsymbol{n}$ & \% \\
\hline $\begin{array}{l}\text { Behandlung in den letzten 12 Monaten } \\
\text { Wenn in Behandlung, Art der Behandlung }\end{array}$ & 2768 & - & 12.348 & - \\
\hline $\begin{array}{l}\text { Schilddrüsenhormone } \\
\text { Radiojodtherapie }\end{array}$ & 2189 & 79,1 & 10.820 & 87,6 \\
\hline Bestrahlung im Halsbereich & 276 & 10,0 & 1022 & 8,3 \\
\hline Schilddrüsenoperation & 66 & 2,4 & 131 & 1,1 \\
\hline Jodtabletten & 825 & 29,8 & 2841 & 23,0 \\
\hline Andere Behandlung & 789 & 28,5 & 3237 & 26,2 \\
\hline
\end{tabular}

Informationen wurden in einem persönlichen Interview erhoben. Erfragt wurde von Teilnehmenden mit Diagnose einer Schilddrüsenerkrankung, wie diese behandelt wurde. "Keine Angabe", "weiß nicht" und unerlaubt fehlende Werte: Summe jeweils $\leq 1,8 \%$

${ }^{2}$ Frage nur gestellt an Teilnehmende, die eine Diagnose einer Schilddrüsenerkrankung und deren Behandlung in den vorangegangenen 12 Monaten angegeben haben. Anteil bezogen auf Teilnehmende mit berichteter Diagnose und Behandlung einer Schilddrüsenerkrankung. Mehrfachnennungen möglich

${ }^{\mathrm{b}}$ Teilnehmende wurden gebeten, auch Behandlungen, die länger als 12 Monate zurückliegen, zu berichten und unbekannter Hypertonie oder Dyslipidämie. Allerdings ist die DEGS-Population mit etwa 8100 Teilnehmenden vergleichsweise klein [8]. GEDA 2012 und 2014/2015 mit 19.300 bzw. 24.000 Teilnehmenden sind reine Telefonsurveys, die die hier untersuchten Erkrankungen als 12-Monats-Prävalenz (Diabetes auch als Lebenszeitprävalenz) erfassen $[14,15]$. Darüber hinaus liegen für Deutschland auch Häufigkeitsschätzungen aus amtlichen Statistiken oder Routinedaten vor, wie aus dem Herzbericht [19], der GBE [21] oder Krankenkassendaten [30, 31]. Diese umfassen zwar meist bundesweite Diagnosestatistiken, der Vergleich mit in der NAKO beobachteten Häufigkeiten selbst berichteter, jemals erfolgter ärztlicher Diagnosen von kardiovaskulären und metabolischen Erkrankungen sowie Operationen ist aber limitiert; zum Beispiel erfasst der Deutsche Herzbericht nur vollstationäre kardiovaskuläre Fälle eines Jahres [19].

Für die Einordnung der beobachteten Häufigkeiten wurden in der vorliegenden Studie altersstandardisierte $\mathrm{Ra}$ ten berechnet. Wie aus vergleichbaren Kohortenstudien bekannt [32,33], deutet sich an, dass die NAKO-Population eine geringere kardiovaskuläre und metabolische Morbidität aufweist als die zugrunde liegende Bevölkerung. Zu berücksichtigen ist dabei, dass die Teilnahmebereitschaft in der NAKO zur Halbzeit der Basiserhebung bei etwa $18 \%$ lag. Daher muss bei der Interpretation berechneter relativer Häufigkeiten berücksichtigt werden, dass diese nicht unmittelbar auf die deutsche Bevölkerung übertragbar sind. Für Kohortenanalysen und die Berechnung von Assoziationen und relativen Risiken stellt diese Tatsache vermutlich kein größeres methodisches Problem dar, muss aber bei der Analyse absoluter und attributaler Risiken sowie bei der Prädiktion berücksichtigt werden. In der großen epidemiologischen UK-Biobank-Studie aus dem Vereinigten Königreich lag die Responseproportion beispielsweise bei nur 5,5\% [34]. Analysen der UK Biobank bestätigen die Annahme, dass die dort beobachteten UrsacheWirkungs-Assoziationen eine gute Generalisierbarkeit für das Erhebungsgebiet erlauben [34]. Für die NAKO ist zu beach- 
Tab. 6 Häufigkeiten von selbst berichteten kardiologischen und gefäßchirurgischen Operationen sowie Schilddrüsenresektionen in der NAKO zu Halbzeit der Basiserhebung

\begin{tabular}{|c|c|c|c|c|c|c|c|c|c|c|}
\hline & \multicolumn{5}{|c|}{ Männer $(n=47.266)$} & \multicolumn{5}{|c|}{ Frauen $(n=54.540)$} \\
\hline & \multicolumn{4}{|c|}{ NAKO } & \multirow{2}{*}{$\begin{array}{l}\text { Vergleichs- } \\
\text { daten } \\
\%\end{array}$} & \multicolumn{4}{|c|}{ NAKO } & \multirow{2}{*}{$\begin{array}{l}\text { Vergleichs- } \\
\text { daten } \\
\%\end{array}$} \\
\hline & $n$ & $\%$, roh & $\begin{array}{l}\%, \\
\text { stand. }^{a}\end{array}$ & $\begin{array}{l}\text { \%, } \\
\text { stand. }\end{array}$ & & $n$ & $\%$, roh & $\begin{array}{l}\%, \\
\text { stand. }^{a}\end{array}$ & $\begin{array}{l}\text { \%, } \\
\text { stand. }\end{array}$ & \\
\hline Herzklappenoperation & 279 & 0,6 & 0,5 & 0,4 & $0,42[19]^{\mathrm{c}}$ & 117 & 0,2 & 0,2 & 0,2 & $0,42[19]^{\mathrm{c}}$ \\
\hline PTCA & 1928 & 4,1 & 2,9 & 2,4 & - & 528 & 1,0 & 0,8 & 0,6 & - \\
\hline Bypassoperation am Herzen & 549 & 1,2 & 0,8 & 0,7 & $0,58[19]^{c}$ & 71 & 0,1 & 0,1 & 0,1 & $0,58[19]^{c}$ \\
\hline Schrittmacherimplantation & 315 & 0,7 & 0,5 & 0,4 & $-[19]^{c d}$ & 140 & 0,3 & 0,2 & 0,2 & $-[19]^{c d}$ \\
\hline $\begin{array}{l}\text { Ballondilatation der Beinar- } \\
\text { terien }\end{array}$ & 386 & 0,8 & 0,5 & 0,5 & - & 126 & 0,2 & 0,2 & 0,1 & - \\
\hline $\begin{array}{l}\text { Bypassoperation an den } \\
\text { Beinarterien }\end{array}$ & 159 & 0,3 & 0,2 & 0,2 & - & 45 & 0,08 & 0,06 & 0,05 & - \\
\hline Karotisangioplastie & 166 & 0,4 & 0,3 & 0,2 & - & 76 & 0,1 & 0,1 & 0,08 & - \\
\hline Schilddrüsenresektion & 1173 & 2,5 & 1,8 & 1,6 & $-[25]^{e}$ & 3676 & 6,7 & 5,2 & 4,5 & $-[25]^{e}$ \\
\hline \multicolumn{11}{|c|}{$\begin{array}{l}\text { Informationen wurden in einem persönlichen Interview erhoben. Erfragt wurde von allen Teilnehmenden anhand einer vordefinierten Liste, ob bei ihnen } \\
\text { jemals eine kardiologische oder gefäßchirurgische Operation oder eine Schilddrüsenresektion durchgeführt wurde. „Keine Angabe“, „weiß nicht” und } \\
\text { unerlaubt fehlende Werte: Summe jeweils } \leq 0,2 \% \text {. PTCA, perkutane transluminale Coronar-Angioplastie (Ballondilatation der Herzkranzgefäße) } \\
\text { 'Direkte Altersstandardisierung basierend auf Zahlen des Statistischen Bundesamtes } 2011 \text { [26] } \\
\text { 'Direkte Altersstandardisierung basierend auf der "alten Europastandardbevölkerung” [26] } \\
\text { 'Häufigkeiten im Deutschen Herzbericht werden pro } 100.000 \text { bzw. } 1.000 .000 \text { Einwohner und hier gerundet in Prozent angegeben; keine geschlechtsstratifi- } \\
\text { zierte Häufigkeit berichtet } \\
\text { 'Der Deutsche Herzbericht geht für } 2017 \text { in Deutschland bezüglich implantierter kardialer Rhythmusimplantate von } 77.370 \text { Schrittmachern bzw. } 25.846 \\
\text { implantierbaren Kardiovertern/Defibrillatoren aus; keine geschlechtsstratifizierte Häufigkeit berichtet } \\
\text { 'Das Statistische Bundesamt geht für } 2017 \text { in Deutschland von rund } 77.000 \text { Schilddrüsenresektionen aus (Summe vollständiger oder partieller Parathyreo- } \\
\text { idektomien); keine geschlechtsstratifizierte Häufigkeit berichtet }\end{array}$} \\
\hline
\end{tabular}

ten, dass sich die Häufigkeitsverteilungen in der finalen Stichprobe noch ändern können, da sich im Verlauf der weiteren Rekrutierung noch Verschiebungen in der Teilnehmerstruktur ergeben können.

Die beobachteten Häufigkeiten beruhen auf Selbstangaben, bei deren Interpretation deren Validität berücksichtigt werden muss. Im Rahmen der europäischen Kohortenstudie European Prospective Investigation into Cancer and Nutrition wurden für Deutschland für die Selbstangabe des Herzinfarktes positiv prädiktive Werte (PPV, Vergleich mit Arztangaben) von $64-67 \%$ angegeben, für Herzinsuffizienz $24 \%$, für arterielle Hypertonie $89 \%$ und für Diabetes 85-94\% [35]. Okura et al. berichten für diese Erkrankungen PPV von $73 \%$, $37 \%, 86 \%$ und $94 \%$, negativ prädiktive Werte von $99 \%, 99 \%, 89 \%$ und $97 \%$, Sensitivitäten von $90 \%, 69 \%, 82 \%$ und $66 \%$ und Spezifitäten von 92-99,7\% [36]. Pastorino et al. berichten für Diabetes einen PPV von 95\% [37], Molenaar et al. für Hypertonie und Diabetes Sen- sitivitäten von $35 \%$ bzw. $59 \%$ und Spezifitäten von $96 \%$ bzw. $99 \%$ [38]. Um selbst berichtete Diagnosen abzusichern bzw. unbekannte Fälle zu identifizieren, sind in der NAKO-Basiserhebung weitergehende Datenaufbereitungen geplant, wie die Einbeziehung klinischer Routineparameter und Untersuchungsmesswerte. Dieser Ansatz wurde in der vorliegenden Studie noch nicht genutzt, was Abweichungen zwischen den hier beobachteten und den Vergleichsdaten zu Häufigkeiten kardiovaskulärer und metabolischer Erkrankungen sowie Operationen mit erklären kann. Auch der Vergleich unterschiedlicher Datenquellen (z. B. Selbstbericht in der NAKO vs. Abrechnungsdaten im Herzbericht [19]), Fragestellungen (z. B. „Verengung der Herzkranzgefäße bzw. Angina pectoris“ in der NAKO vs. , gestörte Blutzufuhr zum Herzen, Verengung der Herzkranzgefäße oder Angina pectoris“ in DEGS [9]) oder Erhebungsmethoden (z.B. Selbstangabe Schilddrüsenerkrankung in der NAKO vs. Sonographiemessung in SHIP [39]) kann Abweichungen er- klären. Ebenso müssen Selbstangaben zu Behandlungsformen vorsichtig interpretiert werden. So gaben rund 5-20\% der NAKO-Teilnehmenden an, beim Herzinfarkt nicht stationär behandelt worden $\mathrm{zu}$ sein. Dies erscheint überraschend, da Herzinfarkte in der Regel stationär behandelt werden. Es bleibt unklar, ob es sich bei den berichteten Fällen um stumme, zufällig entdeckte Infarkte handelt oder gar keine Infarkte zugrunde liegen. Die Einbeziehung von Ergebnissen der kardialen Untersuchungen in der NAKO (EKG, Echo) könnte hier in zukünftigen Analysen aufschlussreich sein.

Stärken unserer Studie sind das multizentrische Design einer bundesweiten populationsbasierten Beobachtungsstudie, die Größe der Population mit mehr als 100.000 Teilnehmenden (bereits jetzt die größte Bevölkerungsstudie in Deutschland) und die standardisierte Datenerhebung mit identischen Methoden unter einer zentralen Qualitätskontrolle. Letzteres ermöglichte die einheitliche Erfassung eines breiten Spektrums an Charakteristika der 
Teilnehmenden. Allerdings weist die im Vergleich etwas geringere Häufigkeit kardiovaskulärer und metabolischer Erkrankungen auf einen Selektionseffekt und damit eine potenziell reduzierte externe Validität (Repräsentativität) hin. So ist für epidemiologische Studien bekannt, dass Teilnehmende tendenziell gesundheitsbewusster sind als die Allgemeinbevölkerung [27]. Darüber hinaus ist aus anderen Studien bekannt, dass die Häufigkeit kardiovaskulärer und metabolischer Erkrankungen in Abhängigkeit vom Sozialstatus und Bundesland variiert [40-43], was in den vorliegenden Analysen noch nicht berücksichtigt wurde. Da die analysierten Daten auf Selbstangaben basieren, können Antwortverzerrungen nicht ausgeschlossen werden, die aufgrund von sozialer Erwünschtheit (Social Bias) oder Erinnerungsfehlern (Reporting Bias) auftreten. Bei der Interpretation der Verteilung des berichteten Alters bei Erstdiagnose bzw. Operation muss zum einen die zugrunde liegende Altersverteilung zum Zeitpunkt des Berichtens berücksichtigt werden, zum anderen, dass die Erkrankung nicht zum Diagnosezeitpunkt, sondern retrospektiv erfragt wurde. Für einige der gezeigten Daten sind uns für Deutschland keine publizierten Vergleichsdaten oder geschlechtsspezifischen Zahlen bekannt, sodass keine bzw. nur eingeschränkte Vergleiche möglich waren.

\section{Fazit}

Mit den vorliegenden Daten liegen Fallzahlen für selbst berichtete kardiovaskuläre und metabolische Erkrankungen und Operationen für die ersten 101.806 NAKO-Teilnehmenden vor. Die beobachteten Häufigkeiten sind vergleichbar mit für Deutschland berichteten Zahlen, wenn auch etwas niedriger. Diese Datenressource unterstreicht die Bedeutung der NAKO für weitergehende Analysen im Bereich der kardiometabolischen Forschung in der deutschen Erwachsenenbevölkerung.

\section{Korrespondenzadresse}

\section{Dr. Lina Jaeschke, PH, MPH}

Forschergruppe Molekulare Epidemiologie, Max-Delbrück-Centrum für Molekulare Medizin in der Helmholtz-Gemeinschaft (MDC)

Robert-Rössle-Straße 10, 13125 Berlin, Deutschland

lina.jaeschke@mdc-berlin.de

Danksagung. Wir danken allen Teilnehmerinnen und Teilnehmern sowie den Mitarbeiterinnen und Mitarbeitern der NAKO Gesundheitsstudie.

Förderung. Dieses Projekt wurde mit Daten der NAKO Gesundheitsstudie durchgeführt (www.nako. de). Die NAKO Gesundheitsstudie wird durch das Bundesministerium für Bildung und Forschung (BMBF, Förderkennzeichen 01ER1301A/B/C und 01ER1511D), die Bundesländer und die HelmholtzGemeinschaft gefördert sowie durch die beteiligten Universitäten und Institute der Leibniz-Gemeinschaft finanziell unterstützt.

Funding. Open Access funding provided by Projekt DEAL.

\section{Einhaltung ethischer Richtlinien}

Interessenkonflikt. L. Jaeschke, A. Steinbrecher, K.H. Greiser, M. Dörr, T. Buck, J. Linseisen, C. Meisinger, W. Ahrens, H. Becher, K. Berger, B. Braun, H. Brenner, S. Castell, B. Fischer, C.-W. Franzke, S. Gastell, K. Günther, W. Hoffmann, B. Holleczek, A. Jagodzinski, R. Kaaks, A. Kluttig, G. Krause, L. Krist, O. Kuß, A.-T. Lehnich, M. Leitzmann, W. Lieb, M. Löffler, K.B. Michels, R. Mikolajczyk, A. Peters, T. Schikowski, S. Schipf, B. Schmidt, M. Schulze, H. Völzke, S.N. Willich und T. Pischon geben an, dass kein Interessenkonflikt besteht.

Alle beschriebenen Untersuchungen am Menschen wurden mit Zustimmung der zuständigen Ethik-Kommission, im Einklang mit nationalem Recht sowie gemäß der Deklaration von Helsinki von 1975 (in der aktuellen, überarbeiteten Fassung) durchgeführt. Von allen Teilnehmenden liegt eine Einverständniserklärung vor.

Open Access. Dieser Artikel wird unter der Creative Commons Namensnennung 4.0 International Lizenz veröffentlicht, welche die Nutzung, Vervielfältigung, Bearbeitung, Verbreitung und Wiedergabe in jeglichem Medium und Format erlaubt, sofern Sie den/die ursprünglichen Autor(en) und die Quelle ordnungsgemäß nennen, einen Link zur Creative Commons Lizenz beifügen und angeben, ob Änderungen vorgenommen wurden.

Die in diesem Artikel enthaltenen Bilder und sonstiges Drittmaterial unterliegen ebenfalls der genannten Creative Commons Lizenz, sofern sich aus der Abbildungslegende nichts anderes ergibt. Sofern das betreffende Material nicht unter der genannten Creative Commons Lizenz steht und die betreffende Handlung nicht nach gesetzlichen Vorschriften erlaubt ist, ist für die oben aufgeführten Weiterverwendungen des Materials die Einwilligung des jeweiligen Rechteinhabers einzuholen.
Weitere Details zur Lizenz entnehmen Sie bitte der Lizenzinformation auf http://creativecommons.org/ licenses/by/4.0/deed.de.

\section{Literatur}

1. Robert Koch-Institut (2015) Gesundheit in Deutschland. Gesundheitsberichterstattung des Bundes. Gemeinsam getragen von RKI und DESTATIS. RKI, Berlin. https://www.destatis.de/ DE/Themen/Gesellschaft-Umwelt/Gesundheit/ Gesundheitszustand-Relevantes-Verhalten/ Publikationen/Downloads-Gesundheitszustand/ gesundheit-in-deutschland-publikation.pdf? blob=publicationFile\&v=3. Zugegriffen: 26. März 2019

2. Plass D, Vos T, Hornberg C, Scheidt-Nave C, Zeeb H Krämer A (2014) Entwicklung der Krankheitslast in Deutschland. Dtsch Arztebl Int 111:629-638

3. Wichmann HE, Kaaks R, Hoffmann W, Jockel KH, Greiser KH, Linseisen J (2012) The German national cohort. Bundesgesundheitsblatt Gesundheitsforschung Gesundheitsschutz 55:781-787

4. National Cohort Consortium G (2014) The German National Cohort: aims, study design and organization. Eur JEpidemiol 29:371-382

5. National Cohort Consortium G (2015) The German National Cohort-A prospective epidemiological study resource for health and disease research in Germany. https://nako.de/wp-content/ uploads/2015/07/Wissenschaftliches-Konzeptder-NAKO2.pdf.Zugegriffen:4. Apr. 2018

6. Schipf S, Schöne G, Schmidt B et al (2020) Die Basiserhebung der NAKO Gesundheitsstudie: Teilnahme an den Untersuchungsmodulen, ihre Qualitätssicherung, und Nutzung von Sekundärdaten. Bundesgesundheitsbl. https://doi.org/10. 1007/s00103-020-03093-z

7. Völzke H, Alte D, Schmidt CO et al (2010) Cohort profile: the study of health in pomerania. Int J Epidemiol 40:294-307

8. Scheidt-Nave C, Kamtsiuris P, Gößwald A et al (2012) German health interview and examination survey for adults (DEGS) — design, objectives and implementation of the first data collection wave. BMC Public Health 12:730-730

9. Gosswald A, Schienkiewitz A, Nowossadeck E, Busch MA (2013) Prevalence of myocardial infarction and coronary heart disease in adults aged 40-79 years in Germany: results of the German Health Interview and Examination Survey for Adults (DEGS1). Bundesgesundheitsblatt Gesundheitsforschung Gesundheitsschutz 56:650-655

10. Neuhauser H, Thamm M, Ellert U (2013) Blutdruck in Deutschland 2008-2011 - Ergebnisse der Studie zur Gesundheit Erwachsener in Deutschland (DEGS1). J Health Monit 56:795-801

11. Heidemann C, Du Y, Paprott R, Haftenberger M, Rathmann W, Scheidt-Nave C (2016) Temporal changes in the prevalence of diagnosed diabetes, undiagnosed diabetes and prediabetes: findings from the German Health Interview and Examination Surveys in 1997-1999 and 2008-2011. Diabet Med 33:1406-1414

12. Heidemann C, Du Y, Schubert I, Rathmann W, Scheidt-Nave C (2013) Prävalenz und zeitliche Entwicklung des bekannten Diabetes mellitus. Bundesgesundheitsblatt Gesundheitsforschung Gesundheitsschutz 56:668-677

13. Scheidt-Nave C, Du Y, Knopf $\mathrm{H}$ et al (2013) Verbreitung von Fettstoffwechselstörungen bei Erwachsenen in Deutschland-Ergebnisse der Stu- 


\section{Leitthema}

die zur Gesundheit Erwachsener in Deutschland (DEGS1). Bundesgesundheitsblatt Gesundheitsforschung Gesundheitsschutz 56:661-667

14. Robert Koch-Institut (2012) Beiträge zur Gesundheitsberichterstattung des Bundes. Daten und Fakten: Ergebnisse der Studie "Gesundheit in Deutschland aktuell 2012". https://www. rki.de/DE/Content/Gesundheitsmonitoring/ Gesundheitsberichterstattung/GBEDownloadsB/ GEDA09.pdf? _blob=publicationFile. Zugegriffen: 12 . Juni 2019

15. Saß A-C, Lange C, Finger JD et al (2017) "Gesundheit in Deutschland aktuell" - Neue Daten für Deutschland und Europa Hintergrund und Studienmethodik von GEDA 2014/2015-EHIS. J Health Monit. https://doi.org/10.17886/RKIGBE-12017-17012

16. Kamtsiuris $P$, Lange $M$, Hoffmann R et al (2013) Die erste Welle der Studie zur GesundheitErwachsener in Deutschland (DEGS1). Bundesgesundheitsblatt 56:611-619

17. Völzke H (2012) Study of Health in Pomerania (SHIP). Konzept, Kohortendesign und ausgewählte Ergebnisse. Bundesgesundheitsblatt Gesundheitsforschung Gesundheitsschutz 55:790-794

18. Raum P, Lamparter J, Ponto KA et al (2015) Prevalence and cardiovascular associations of diabetic retinopathy and maculopathy: results from the Gutenberg health study. PLoS ONE 10:e127188-e127188

19. Deutsche Herzstiftung (2018) Deutscher Herzbericht 2018. DeutscheHerzstiftunge. V.Frankfurtam Main. https://www.herzstiftung.de/herzbericht. Zugegriffen: 6. Juni 2019

20. Ohlmeier C, Mikolajczyk R, Frick J, Prütz F, Haverkamp W, Garbe E (2015) Incidence, prevalence and 1-year all-cause mortality of heart failure in Germany: a study based on electronic healthcare data of more than six million persons. Clin Res Cardiol 104:688-696

21. Robert Koch-Institut (1998) Gesundheitsbericht für Deutschland, 1998. Kapitel 5.4 Periphere arterielle Verschlußkrankheit. Gesundheitsberichterstattung des Bundes - Gemeinsam getragen von RKI und DESTATIS. http://www. gbe-bund.de/gbe10/abrechnung.prc_abr_test_ logon?p_uid=gast\&p_aid=0\&p_knoten $=$ FID \&p sprache=D\&p_suchstring=896. Zugegriffen: 12 . Juni 2019

22. Deutsche Diabetes Gesellschaft (2019) Deutscher Gesundheitsbericht. Diabetes 2019. Die Bestands aufnahme. Deutsche Diabetes Gesellschaft (DDG) und diabetesDE - Deutsche Diabetes-Hilfe. Verlag Kirchheim + Co GmbH, Mainz. https://www. deutsche-diabetes-gesellschaft.de/fileadmin/ Redakteur/Stellungnahmen/Gesundheitspolitik/ 20181114gesundheitsbericht_2019.pdf. Zugegriffen: 5 . Juni 2019

23. Deutsche Gicht-Liga (2019) http://www.gichtliga. de/home.html.Zugegriffen:7. Juni 2019

24. Deutsche Rheuma-Liga (2019) https://www. rheuma-liga.de/gicht/.Zugegriffen:7. Juni 2019

25. Statistisches Bundesamt (Destatis) (2017) Fallpauschalenbezogene Krankenhausstatistik (DRG-Statistik) - Operationen und Prozeduren der vollstationären Patientinnen und Patienten der Krankenhäuser (4-Steller). https://www. destatis.de/DE/Themen/Gesellschaft-Umwelt/ Gesundheit/Krankenhaeuser/Publikationen/ Downloads-Krankenhaeuser/operationenprozeduren-5231401177015.html. Zugegriffen: 12. Juni 2019

26. Robert Koch-Institut (2019) Informationssystem der Gesundheitsberichterstat- tung des Bundes. http://www.gbe-bund. de/gbe10/ergebnisse.prc_tab?fid $=10216 \&$ suchstring=standardbev\%F6lkerung\&query $\mathrm{id}=\&$ sprache $=$ D\&fund_typ $=$ DEF \&methode $=2 \&$ $\mathrm{vt}=1 \&$ verwandte $=1 \&$ page_ret $=0 \&$ seite $=\& p \_$_lfd $\mathrm{nr}=2 \& p \_n e w s=\& p \_s p r a c h k z=D \& p \_u i d=$ gast $\&$ p_aid $=97016022 \& h l p \_n r=3 \& p \_j a n e i n=J \#$ SEARCH $=\% 2522$ standardbev\%C3\%B6lkerung \%2522.Zugegriffen:21.Juni 2019

27. Ahrens W, Pigeot I (2005) Handbook of epidemiology. Springer, Berlin Heidelberg, S 143-145

28. Heidemann C, Scheidt-Nave C (2017) Prävalenz, Inzidenz und Mortalität von Diabetes mellitus bei Erwachsenen in Deutschland - Bestandsaufnahme zur Diabetes-Surveillance. J Health Monit. https://doi.org/10.17886/RKI-GBE-12017-17050

29. Heidemann C, Kuhnert R, Born S, Scheidt-Nave C (2017) 12-Monats-Prävalenz des bekannten Diabetes mellitus in Deutschland. J Health Monit 2:48-56

30. Goffrier BSM, Bätzing-Feigenbaum J (2017) Administrative Prävalenzen und Inzidenzen des Diabetesmellitusvon 2009bis 2015.Zentralinstitut für die kassenärztliche Versorgung in Deutschland (Zi). Versorgungsatlas-Bericht Nr. 17/03. Berlin. https://www.versorgungsatlas.de/themen/alleanalysen- nach-datum-sortiert/?tab=6\&uid $=79$. Zugegriffen:23. Aug. 2019

31. Tamayo T, Brinks R, Hoyer A, Kuß O, Rathmann W (2016) The prevalence and incidence of diabetes in Germany: an analysis of statutory health insurance data on 65 million individuals from the years 2009 and 2010. Dtsch Arztebl Int 113:177-182

32. Boeing H, Korfmann A, Bergmann MM (1999) Recruitment procedures of EPIC-Germany. European Investigation into Cancer and Nutrition. Ann Nut Metab 43:205-215

33. Fry A, Littlejohns TJ, Sudlow C et al (2017) Comparison of sociodemographic and healthrelated characteristics of UK Biobank participants with those of the general population. Am J Epidemiol 186:1026-1034

34. Batty GD, Gale C, Kivimaki M, Deary I, Bell S (2019) Generalisability of results from UK Biobank: comparison with a pooling of 18 cohort stu dies. medRxiv. https://doi.org/10.1101/19004705: 19004705

35. Herrmann WJ, Weikert C, Bergmann $M$ et al (2018) Erfassung inzidenter kardiovaskulärer und metabolischer Erkrankungen in epidemiologischen Kohortenstudien in Deutschland. Bundesgesundheitsblatt Gesundheitsforschung Gesundheitsschutz 61:420-431

36. Okura Y, Urban LH, Mahoney DW, Jacobsen SJ, Rodeheffer RJ (2004) Agreement between selfreport questionnaires and medical record data was substantial for diabetes, hypertension, myocardial infarction and stroke but not for heart failure. J Clin Epidemiol 57:1096-1103

37. Pastorino S, Richards M, Hardy R et al (2015) Validation of self-reported diagnosis of diabetes in the 1946 British birth cohort. Prim Care Diabetes 9:397-400

38. Molenaar EA, Ameijden EJCV, Grobbee DE, Numans ME(2006) Comparison of routine care selfreported and biometrical data on hypertension and diabetes: results of the Utrecht Health Project. Eur J Public Health 17:199-205

39. Völzke H, Thamm M (2007) Epidemiologie von Schilddrüsenerkrankungen in Deutschland. Präv Gesundheitsf 2:149-152

40. Robert Koch-Institut (2016) Gesundheit in Deutschland - die wichtigsten Entwicklungen. Gesundheitsberichterstattung des Bundes.
Gemeinsam getragen von RKI und DESTATIS. RKI, Berlin. https://www.rki. de/DE/Content/Gesundheitsmonitoring/ Gesundheitsberichterstattung/GBEDownloads GiD/2015/kurzfassung_gesundheit_in_ deutschland.pdf? _ blob=publicationFile. Zugegriffen: 26.Okt. 2019

41. Dornquast C, Kroll LE, Neuhauser HK, Willich SN, Reinhold T, Busch MA (2016) Regionale Unterschiede in der Prävalenz kardiovaskulärer Erkrankungen. Dtsch Arztebl Int 113:704-711

42. Schipf S, Werner A, Tamayo T et al (2012) Regional differences in the prevalence of known Type 2 diabetes mellitus in 45-74 years old individuals: results from six population-based studies in Germany (DIAB-CORE Consortium). Diabet Med 29:e88-e95

43. Diederichs C, Neuhauser H (2014) Regional variations in hypertension prevalence and management in Germany: results from the German Health Interview and Examination Survey (DEGS1).JHypertens 32:1405-1414 


\section{Affiliations}

${ }^{1}$ Forschergruppe Molekulare Epidemiologie, Max-Delbrück-Centrum für Molekulare Medizin in der Helmholtz-Gemeinschaft (MDC), Berlin, Deutschland; ${ }^{2}$ Abteilung Epidemiologie von Krebserkrankungen, Deutsches Krebsforschungszentrum (DKFZ), Heidelberg, Deutschland; ${ }^{3}$ Institut für Community Medicine, Universitätsmedizin Greifswald, Greifswald, Deutschland; ${ }^{4}$ Partnerstandort Greifswald, Deutsches Zentrum für Herz-Kreislauf-Forschung (DZHK), Greifswald, Deutschland; ${ }^{5}$ Klinikum Westfalen, Knappschaftskrankenhaus Dortmund, Dortmund, Deutschland; ${ }^{6}$ Lehrstuhl für Epidemiologie,

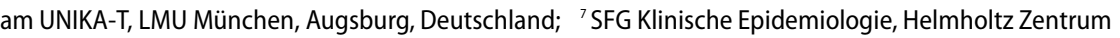
München, Neuherberg, Deutschland; ${ }^{8}$ Leibniz-Institut für Präventionsforschung und Epidemiologie BIPS, Bremen, Deutschland; ${ }^{9}$ Institut für Statistik, Universität Bremen, Bremen, Deutschland; ${ }^{10}$ Institut für Medizinische Biometrie und Epidemiologie, Universitätsklinikum Hamburg-Eppendorf, Hamburg, Deutschland; "Institut für Epidemiologie und Sozialmedizin, Westfälische Wilhelms-Universität Münster, Münster, Deutschland; ${ }^{12}$ Institut für Krebsepidemiologie an der Universität zu Lübeck, Lübeck, Deutschland; ${ }^{13}$ Abteilung Klinische Epidemiologie und Alternsforschung, Deutsches Krebsforschungszentrum (DKFZ), Heidelberg, Deutschland; ${ }^{14}$ Abteilung Epidemiologie, HelmholtzZentrum für Infektionsforschung (HZl), Braunschweig, Deutschland; ${ }^{15}$ Institut für Epidemiologie und Präventivmedizin, Universität Regensburg, Regensburg, Deutschland; ${ }^{16}$ Institut für Prävention und Tumorepidemiologie, Universitätsklinikum Freiburg, Medizinische Fakultät, Albert-Ludwigs-Universität Freiburg, Freiburg, Deutschland; ${ }^{17}$ Zentrale Einrichtung NAKO-Studienzentrum, Deutsches Institut für Ernährungsforschung (DIfE), Potsdam-Rehbrücke, Deutschland; ${ }^{18}$ Krebsregister Saarland, Saarbrücken, Deutschland; ${ }^{19}$ Institut für Medizinische Epidemiologie, Biometrie und Informatik, Martin-LutherUniversität Halle-Wittenberg, Halle (Saale), Deutschland; ${ }^{20}$ Medizinische Hochschule Hannover (MHH), Hannover, Deutschland; ${ }^{21}$ Institut für Sozialmedizin, Epidemiologie und Gesundheitsökonomie, Charité Universitätsmedizin Berlin, Berlin, Deutschland; ${ }^{22}$ Institut für Biometrie und Epidemiologie, Deutsches Diabetes-Zentrum (DDZ), Leibniz-Zentrum für Diabetes-Forschung an der Heinrich-Heine-Universität Düsseldorf, Düsseldorf, Deutschland; ${ }^{23}$ Institut für Medizinische Informatik, Biometrie und Epidemiologie, Universität Duisburg-Essen, Essen, Deutschland; ${ }^{24}$ Institut für Epidemiologie, Christian-AlbrechtsUniversität Kiel, Kiel, Deutschland; ${ }^{25}$ Institut für Medizinische Informatik, Statistik und Epidemiologie (IMISE), Medizinische Fakultät, Universität Leipzig, Leipzig, Deutschland; ${ }^{26}$ Institut für Epidemiologie, Helmholtz Zentrum München - Deutsches Forschungszentrum für Gesundheit und Umwelt, Neuherberg, Deutschland; ${ }^{27}$ IUF Leibniz-Institut für umweltmedizinische Forschung $\mathrm{gGmbH}$, Düsseldorf, Deutschland; ${ }^{28}$ Standort Greifswald, Deutsches Zentrum für Diabetesforschung (DZD), Greifswald, Deutschland;

${ }^{29}$ Abteilung Molekulare Epidemiologie, Deutsches Institut für Ernährungsforschung (DIfE), PotsdamRehbrücke, Deutschland; ${ }^{30}$ Charité - Universitätsmedizin Berlin, Berlin, Deutschland; ${ }^{31}$ Partnerstandort Berlin, Deutsches Zentrum für Herz-Kreislauf-Forschung (DZHK), Berlin, Deutschland; ${ }^{32} \mathrm{MDC} / \mathrm{BIH}$ Biobank, Max-Delbrück-Centrum für Molekulare Medizin in der Helmholtz-Gemeinschaft (MDC) und Berlin Institute of Health (BIH), Berlin, Deutschland 